\title{
In memory of Professor Virgilio Gallai
}

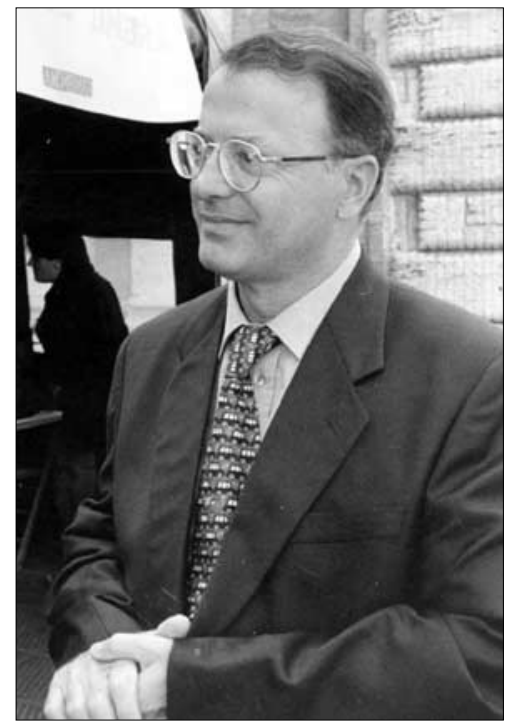

On 26 January 2004, Professor Virgilio Gallai passed away after struggling for the past two years with a tremendous disease. It overcame him despite his courage and determined fight, which drove him to participate in the life of his clinic and in the organization of national and international scientific events until just a few days before his death. This determination and courage were the lite motif of his life, letting him and his group obtain important goals in various fields of neurology.

His interest for headaches began more than 25 years ago with the creation of the Interuniversity Center for the Study of Headache and Neurotransmitter Disorders, which over the years, in addition to Perugia, has involved other centers of national importance in the field of headaches: the Universities of Rome, Bari, Sassari, Naples, Florence, Siena and Modena.

I remember the first time that I met Professor Gallai in 1990. At that moment I was working at the Faculty of Pharmacology at the University of Perugia, and he invited me to join his group. He expressed an urgent need to create a permanent neurochemical laboratory at his clinic that would be dedicated to the study of biochemical and neurotransmitter modifications in patients with headache. He asked me if I could help him reach this objective. He told me that this field was in great expansion and that these studies would give fundamental results, even if, according to him the difficulties of setting up reliable experimental models and of studying patients during headache attacks would represent major obstacles. I accepted the challenge and since then I have always followed his advice and encouragement. Together with Prof. Giovanni Mazzotta, Dr. Andrea Alberti and others who have collaborated with Professor Gallai over the years, I have been able to contribute to the vast research field concerning the pathogenetic mechanisms of headache, and in particular, migraine.

The research carried out on the nutritional state of migraineurs should be remembered in primis: the observation of a reduction of magnesium levels in red and white blood cells of migraine patients suggested a systemic reduction of this element and confirmed the results of ${ }^{31} \mathrm{P}$ magnetic resonance spectroscopy. These data indirectly supported the hypothesis that a condition of neuronal hyperexcitability which contributes to pathophysiological ground of susceptibility to attacks in migraineurs. The hypothesis was confirmed, at the peripheral level, by Professor Gallai's group in an electromyographic study on neuromuscular hyperexcitability during ischemia.

In the wake of evidence that migraine patients are hypersensitive to nitric oxide (NO), Professor Gallai urged me to set up a model to study endogenous metabolism and markers of glutamatergic and serotonergic systems. This led to the development of the platelet model for the study of nitric oxide synthase (NOS) and the intracellular messenger cyclic guanosine monophospate (cGMP) in migraine patients, tested outside and during attacks. Subsequent studies carried out in patients with chronic daily headache demonstrated a correlation between the permanent NO metabolites, nitrites, in cerebrospinal fluid (CSF) and the various glutamatergic and serotoninergic systems. 
A more recent interest shared with Professor Gallai was the role of neurotrophins in patients with chronic daily headache. This led us to measure CSF levels of nerve growth factor and brain-derived neurotrophic factor in relation to glutamate and nitrite levels, to study the sensory neuropeptides calcitonin gene-related peptide, substance $\mathrm{P}$ and neurokinin $\mathrm{A}$, and subsequently to focus on glial cell-line-derived neurotrophic factor in association with somatostatin levels. These studies have provided important insights on the participation of trophic factors, not only in chronic head pain but also in other pathologies characterized by chronic pain, such as fibromyalgia.

Professor Gallai urged me to investigate the events of a headache attack by studying the jugular blood of migraine patients during the critical period. This effort led us to demonstrate the temporal relationships among the early production of nitric oxide metabolites and neuropeptides of trigeminal origin, the delayed production of prostaglandins with vasoactive and algogenic proporties, and more recently, the transitory production of cytokines and adhesion molecules and the upregulation of NFKB. These elements correspond to the activation of both endothelial and inducible forms of NOS during the migraine attack.

Professor Gallai's interests were not limited to the study of biochemical and neurotransmitter modifications of headaches. Other studies were briskly promoted in these later years, especially in the field of headaches typically involving the clinical aspects. In particular, epidemiological studies conducted on pediatric, adult and elderly headache patients, the studies on the relation between headache and sleep, and those on the applicability of the International Headache Society (IHS) classification criteria should be remembered. Finally, in the last months of his life, he promoted studies on the applicability of the diagnostic criteria of the second edition of the International Classification of Headache Disorders (ICHD) through the aid of custom-made software. The other members of Professor Gallai's group and I intend to continue these studies with the enthusiasm that he has always transmitted.

The fervour of activity that characterized his clinical and scientific activity allowed him in little time to obtain a prominent role in the field of headaches in Italy, and also brought international recognition for his scientific activity. With the force and tenacity of his activity, Professor Gallai contributed to the development and success of the Italian Society for the Study of Headaches (SISC), of which he was President from January 2000 to June 2002. During his Presidency, membership tripled to more than 800 , placing SISC among the most important national headache societies, and second in size after the American Headache Society.

His interest in headaches peaked with the foundation of The Journal of Headache and Pain, official journal of SISC and the Italian Society of Pain Clinicians, of which he was Editor-in-Chief until his death. Professor Gallai was also President of the Ad Hoc Committee for the Diagnostic and Therapeutic Guidelines for Migraine and Cluster Headache, whose activity produced a volume in Italian and a version in English, the latter published in The Journal of Headache and Pain in 2001.

Together with Prof. Luigi Alberto Pini, Professor Gallai perceived the urgency of writing a text in Italian dedicated exclusively to headaches not only for specialists in the field, but also for those who were interested in furthering their understanding of headaches or who had to deal with headache pathology in daily practice. Because of this interest, a treatise on headaches was published in 2002, with the contributions of numerous Italian experts in the field.

His undeniable professional qualities and intense scientific activity carried out in the field of headaches, attested to by the numerous articles published in international journals, reached their maximum recognition with the selection of Professor Virgilio Gallai as CoChairman, with Professor Giuseppe Nappi, of the XI Congress of the International Headache Society (IHS), held in Rome on 13-16 September 2003. The Congress was a great success not only because of the presence of so many headache experts, but also because it reached the highest level of participation of all IHS congresses. He also recently played an active role and driving force behind the constitution of the Italian Society for the Study of Stroke, of which in 2003 he became President.

If Professor Gallai's scientific efforts are indisputable, not only in the context of headaches and stroke, but also dementia and multiple sclerosis, the man Gallai was not easy. His life was the clinic, his research, his university duties. He would arrive at the Institute early in the morning and work ceaselessly all day; he also came to the Institute on Sundays and this surely determined our incessant work rhythm. He was a difficult man, of few words, strict, who essentially wanted the most from every one of his collaborators and from all those who were involved with him in projects or in common objectives, university or otherwise. Wanting the most from us not only represented the proof of an agreed upon and confirmed trust, but also denoted a gesture of affection on his part; we were his scientific sons and daughters and from one's "family" one always wants the most! 
There was always an open dialectic with me, a true challenge, but this dialectic motivated me to go ahead and search for new roads.

The members of his team were not the only scientific "pupils", there were many in Italy and abroad who he encouraged to dedicate themselves to headaches and who are now slowly asserting themselves in this field.

Professor Michel Ferrari, past-President of IHS, said of him in a message received immediately after his death: "Professor Gallai was a remarkable man and he will always remain in our memory." It is true that his premature death, after only a few days from the passing of another prominent Italian in the neurological and headache world, Prof. Francomichele Puca, has left an enormous vacancy. Professor Gallai's message will always remain with us: to continue to be united, to work for a common objective and to maintain that uncontaminated passion that must accompany and assist every investigator in his activity and that, only if nurtured and cultivated, can make sense of our professional life.

Acknowledgements Many thanks to John A. Toomey and Marisa M. Morson for editing the English.

Paola Sarchielli Department of Neuroscience, Neurologic Clinic University of Perugia Via Enrico dal Pozzo, I-06126 Perugia, Italy E-mail: headache@unipg.it 